\title{
Contribution à l'optimisation économique des réseaux arborescents de transport de l'eau. Dimensionnement d'un organe hydraulique
}

\author{
Contribution to the economic optimization \\ of water transport branched networks. \\ Dimensioning of a hydraulic section
}
P. Berthomé
P. Bourgine
M. Ezerzer
Y. Penadille
CEMAGREF - Groupement d'Aix-en-Provence

On propose ici quelques possibilités d'utilisation d'une méthode de recherche du dimensionnement optimal d'un organe hydraulique pouvant s'intégrer dans l'optimisation économique des réseaux arborescents de transport de l'eau sous pression ou à surface libre.

Outre les fondements théoriques, des applications pratiques sont présentées concernant uniquement le stade du projet, c'est-à-dire lorsque le tracé en plan est fixé par le projeteur.

La méthode a été utilisée au CEMAGREF pour concevoir un logiciel de dimensionnement des réseaux parcellaires de micro-irrigation.

A method of optimization is proposed for the calculation of a branched network section used for water transport with pressure pipes or open channels.

Theory is detailed with practical applications at the level of the project, i.e. after the choice of the lay-out by the designer.

The CEMAGREF has used this method to develop a computer program for the design of trickle irrigation field systems.

\section{Introduction}

Les réseaux de transport de l'eau sont généralement arborescents : l'eau s'écoule par un seul chemin de "l'origine " du réseau jusqu'aux extrémités (réseau de distribution) ou des extrémités jusqu'à l'origine (réseau de collecte). Cet article n'envisage pas le cas des réseaux maillés, où il $\mathrm{y}$ a plusieurs chemins possibles, et traite uniquement des réseaux de distribution, bien que la méthodologie présentée par la suite s'applique aussi aux réseaux de collecte.

Les réseaux de distribution arborescents sont constitués d'organes hydrauliques résultant d'une décomposition plus ou moins arbitraire effectuée par le projeteur. Ils sont très employés en irrigation sous pression (aspersion et micro-irrigation), ou à surface libre (irrigation de surface). Dans ce cas, les organes hydrauliques sont par exemple des conduites ou bien des canaux. Les organes hydrauliques sont hiérarchisés en organes principaux, primaires, secondaires, etc.

L'optimisation économique d'un réseau de distribution arborescent comprend :

- le choix du meilleur avant-projet possible, l'avantprojet étant défini comme l'ensemble des décisions relatives au tracé du réseau, aux assemblages d'éléments hydrauliques et aux gammes technologiques auxquelles appartiennent ces éléments;

- la recherche du projet de coût minimal, le projet regroupant par définition l'ensemble des décisions de dimensionnement à l'intérieur des gammes définies au niveau de l'avant-projet. 


\section{Liste des symboles}

a Fruit intérieur d'un canal

$b \quad$ Coefficient caractéristique d'une série de diamètres

e Espacement des goutteurs

$f \quad$ Fonction caractéristique de perte de charge

$g \quad$ Fonction de perte de charge

$h \quad$ Cote piézométrique

$h_{0}\left(h_{L}\right) \quad$ Cote imposée à l'origine (à l'abscisse $L$ )

$h^{\min }\left(h^{\max }\right)$ Cote minimale (maximale) imposée

$i, j, k \quad$ Indices

$l \quad$ Largeur au plafond d'un canal

$m \quad$ Coefficient du débit (loi de perte de charge)

$p \quad$ Fonction prix au mètre linéaire d'une conduite

$q \quad$ Débit par unité de longueur

$q_{g} \quad$ Débit nominal unitaire d'un goutteur

$r, s \quad$ Indices

$t \quad$ Tirant d'eau d'un canal

$u \quad$ Variable de dimensionnement (ou de commande)

$x \quad$ Abscisse longitudinale

$z \quad$ Cote du T.N. (terrain naturel)

C Coût unitaire de l'organe hydraulique

$D_{x} \quad$ Domaine de contrainte sur la vitesse

$E(0-L) \quad$ Entonnoir physique

$G \quad$ Gamme technologique

$H^{\min }\left(H^{\max }\right)$ Contrainte équivalente basse (haute)

\begin{tabular}{|c|c|}
\hline$I^{\min }\left(I^{\max }\right)$ & $\begin{array}{l}\text { Pente minimale (maximale) de la ligne piézo- } \\
\text { métrique }\end{array}$ \\
\hline$K_{g}$ & $\begin{array}{l}\text { Coefficient de la loi débit/pression d'un } \\
\text { goutteur }\end{array}$ \\
\hline$K_{s}$ & Coefficient de Strickler \\
\hline$L$ & Longueur de l'organe hydraulique \\
\hline$L_{D E}$ & Largeur d'emprise \\
\hline$L_{R V}$ & Largeur de revêtement \\
\hline$Q$ & Débit \\
\hline$S$ & Section mouillée \\
\hline$S_{D B}$ & Section de déblai \\
\hline$S_{R B}$ & Section de remblai \\
\hline$V$ & Vitesse moyenne \\
\hline$V^{\min }\left(V^{\max }\right)$ & Vitesse minimale (maximale) \\
\hline C & Coût de l'organe hydraulique \\
\hline $\mathbf{H}$ & Fonction Hamiltonien \\
\hline$\alpha$ & $\begin{array}{l}\text { Exposant de la loi débit-pression d'un gout- } \\
\text { teur }\end{array}$ \\
\hline$\beta_{k}$ & $\begin{array}{l}\text { Coefficients caractéristiques d'une gamme } \\
\text { discontinue }\end{array}$ \\
\hline$\phi\left(\phi_{i}\right)$ & Diamètre (d'une gamme discontinue) \\
\hline$\xi$ & Abscisse longitudinale \\
\hline$\pi_{1}$ & Prix du remblai \\
\hline$\pi_{2}$ & Prix du déblai \\
\hline$\pi_{3}$ & Prix du décapage et d'achat d'emprise \\
\hline$\pi_{4}$ & Prix du revêtement \\
\hline$\psi$ & Variable auxiliaire \\
\hline$\psi_{0}\left(\psi_{L}\right)$ & $\begin{array}{l}\text { Valeur de la variable auxiliaire à l'origine (à } \\
\text { l'abscisse } L \text { ) }\end{array}$ \\
\hline
\end{tabular}

On ne traitera ici que de l'optimisation économique au niveau du projet. Elle peut se représenter par un problème de programmation dynamique, qui se résoud à l'aide de la méthode de Labye (Labye et Lechapt, 1961). Cette dernière est actuellement utilisée pour le calcul des réseaux sous pression au CEMAGREF où des logiciels de calcul ont été conçus il y a une dizaine d'années (CEMAGREF, 1983). Mais la méthode de Labye est cependant mal adaptée au calcul des projets de réseaux parcellaires de micro-irrigation (Bourgine, 1982) et de réseaux à surface libre (Ezerzer, 1984).

Nous proposons une autre méthode de calcul appelée $\varepsilon$-optimisation qui consiste à construire un nombre limité de lignes piézométriques de coût minimal sur la totalité du réseau en utilisant le principe du minimum de Pontriaguine, de façon à obtenir une solution approchée de la solution optimale. Cette méthode peut s'appliquer aussi bien aux réseaux sous pression qu'aux réseaux à surface libre et elle a servi de base à la conception d'un logiciel de dimensionnement des réseaux parcellaires de micro-irrigation (CEMAGREF, 1982).

L'objet de cet article est de présenter l' $\varepsilon$-optimisation en se limitant au dimensionnement d'un organe hydraulique.

\section{Hypothèses de base et existence d'une solution hydraulique}

\subsection{Hypothèses de base}

\subsubsection{Données de projet}

- $L:$ longueur de l'organe hydaulique;

$-x$ : abscisse longitudinale (orientation positive dans le sens de l'écoulement de l'eau);

$-z(x)$ : cote du T.N. (terrain naturel) à l'abscisse $x$;

$-Q(x)$ : débit à l'abscisse $x$.

\subsubsection{Géométrie de l'organe}

La section d'abscisse $x$ est caractérisée par une variable de dimensionnement notée $u(x)$, à une ou plusieurs composantes. Par exemple :

- conduite circulaire sous pression : $u=\phi$ où $\phi$ représente le diamètre intérieur;

- canal trapézoïdal : $u=(l, t)$ où $l$ représente la largeur au plafond et $t$ le tirant d'eau (cf. figure 1 ). 
Les caractéristiques géométriques qui ne sont pas retenues comme variable de dimensionnement sont fixées par le projeteur (par exemple $a$, le fruit intérieur du canal, à la figure 1 ).

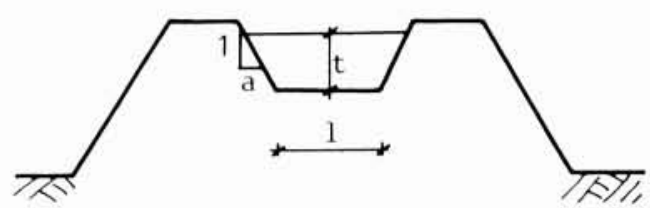

1 . Section-type d'un canal trapézoidal.

\subsubsection{Gamme technologique}

La variable de dimensionnement $u$ appartient à une gamme technologique $G$. La gamme est dite continue si toutes les composantes de $u$ peuvent prendre n'importe quelle valeur dans des intervalles imposés par le projeteur (pour des raisons de construction dans le cas des canaux par exemple). Elle est dite discontinue si une composante au moins de $u$ ne peut prendre qu'un nombre fini de valeurs (diamètres commerciaux de conduites ou gabarits de construction des canaux).

\subsubsection{Contraintes de vitesse}

La vitesse moyenne $V$ doit être comprise entre une valeur minimale $V^{\min }$ (pour éviter les dépôts) et une valeur maximale $V^{\max }$ (pour éviter l'érosion ou la cavitation). On définit ainsi pour chaque abscisse un domaine de contrainte $D$, sur la variable de dimensionnement $u(x)$.

$$
D_{x}=\left\{u / V^{\min } \leqslant V\left(=\frac{Q(x)}{S(u)}\right) \leqslant V^{\max }\right\}
$$

où $S(u)$ représente la section mouillée.

\subsubsection{Coût de l'organe}

Le coût $\mathbf{C}$ de l'organe s'exprime comme suit :

$$
\mathbf{C}=\int_{0}^{L} C\{x, z(x), h(x), u(x)\} \cdot d x
$$

où $C$ représente le coût unitaire de l'organe qui est fonction de l'abscisse $x$ et des valeurs correspondantes de la cote du T.N. $z$, de la cote piézométrique $h$ et de la variable de dimensionnement $u$.

\subsubsection{Perte de charge}

Compte tenu de l'orientation de l'axe des abscisses (positif dans le sens de l'écoulement de l'eau), on peut définir une fonction perte de charge de la forme :

$$
\frac{d h}{d x}(x)=+g\{x, u(x)\}=-Q^{m}(x) \cdot f\{u(x)\}
$$

où $m$ et $f$ désignent un exposant et une fonction caractéristiques de l'organe, et $h$ la cote piézométrique.
En théorie, l'équation $\{1.3\}$ est valable si $h$ représente la charge et non la cote piézométrique (cf. figure 2). On a négligé la variation du terme cinétique en $V^{2}$ dans une première approche, mais la méthode d'optimisation présentée par la suite pourrait être amendée dans ce sens. Cela conduit cependant à une augmentation du temps de calcul disproportionnée avec le gain de précision.

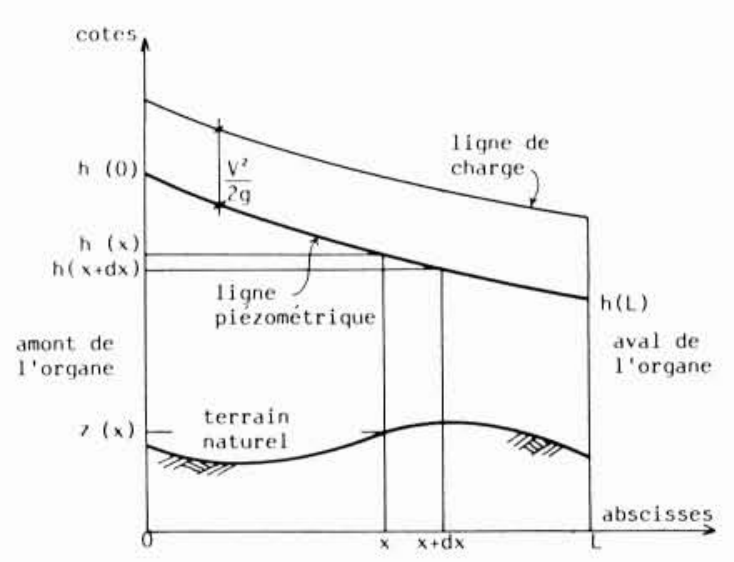

2. Schéma récapitulatif des notations et conventions hydrauliques.

\subsubsection{Contraintes sur la cote piézométrique}

On peut définir un certain nombre de contraintes sur la cote piézométrique $h$, de façon par exemple à éviter les dépassements de pression nominale ou la cavitation dans les conduites, ou bien des dénivelées trop importantes en remblai ou en déblai pour les canaux.

Nous avons retenu une technique approchée par discrétisation consistant à sélectionner un nombre restreint d'abscisses contraignantes, en ignorant les contraintes en dehors de ces abscisses. On dit qu'il y a $\varepsilon$-satisfaction des contraintes continues, le degré de précision $(\varepsilon)$ dépendant du nombre d'abscisses retenues (Bourgine, 1982).

En chaque abscisse contraignante $x$, de l'organe hydraulique, on peut alors écrire :

$$
h^{\min }\left(x_{s}\right) \leqslant h\left(x_{s}\right) \leqslant h^{\max }\left(x_{s}\right)
$$

où $h^{\min }\left(x_{\mathrm{s}}\right)$ et $h^{\max }\left(x_{\mathrm{s}}\right)$ désignent les cotes piézométriques minimale et maximale à l'abscisse $x_{\text {s }}$ (cf. figure 3 ).

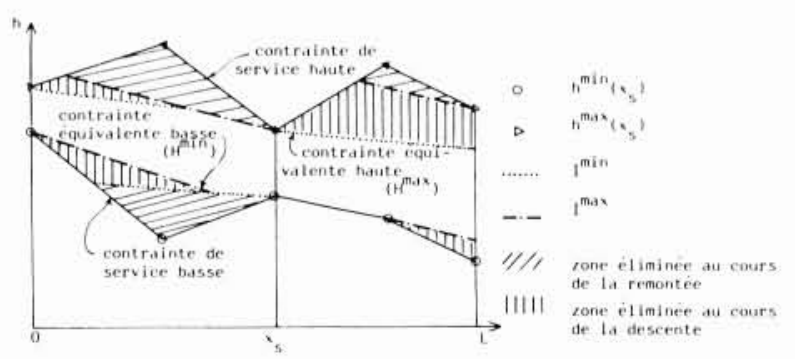

3. Construction de l'entonnoir physique pour un organe hydraulique. 
On définit ainsi une contrainte de service basse (respectivement haute) composée de segments de droite joignant les cotes $h^{\min }\left(x_{s}\right)$ (resp. $h^{\max }\left(x_{s}\right)$ ).

\subsection{Existence d'une solution hydraulique}

Avant de rechercher des solutions optimales, il est nécessaire de vérifier s'il existe au moins une solution hydraulique. Une technique possible consiste à construire les deux lignes piézométriques minimale et maximale respectant les contraintes de service définies précédemment (Bourgine, 1982).

On définit tout d'abord en chaque abscisse $x$ d'un organe donné la pente minimale $I^{\min }$ (resp. maximale $I^{\max }$ ) par :

$$
I^{\min }(x)=\operatorname{Min}_{u}|g\{x, u\}|\left(\text { resp. } I^{\max } \text { et } \operatorname{Max}_{u}\right. \text { ) }
$$

avec $u \in G \cap D_{x}$

La ligne piézométrique basse $H^{\min }$ (resp. haute $H^{\max }$ ) se construit de la façon suivante (cf. figure 3) :

- on " remonte " de l'aval vers l'amont en déterminant les lignes piézométriques de pente $I^{\min }$ (resp. $I^{\max }$ ) dont l'origine aval est un point contraignant $x_{s}$ (cf. $\S 1.1 .7$ ) de cote $h^{\min }\left(x_{s}\right)$ (resp. $h^{\max }\left(x_{s}\right)$ ). On élimine ainsi tous les points du plan situés au-dessous (resp. au-dessus) de ces lignes piézométriques et l'on définit une nouvelle série de points contraignants (comprenant une partie des points contraignants initiaux);

- puis on "descend" de l'amont vers l'aval en construisant à partir de ces points les lignes piézométriques de pente $I^{\max }$ (resp. $I^{\min }$ ) et en éliminant tous les points du plan situés au-dessous (resp. au-dessus) de ces lignes.

La ligne piézométrique basse $H^{\min }$ (resp. haute $H^{\max }$ ) est appelée contrainte équivalente basse (resp. haute). Les contraintes équivalentes basse et haute délimitent un entonnoir physique $E(0-L)$ à l'intérieur duquel sont comprises les lignes piézométriques respectant les différentes contraintes pour l'organe considéré.

La morphologie de l'entonnoir physique permet alors au projeteur de déceler les contradictions, c'est-à-dire les points où la contrainte équivalente basse $H^{\mathrm{min}}$ se trouve au-dessus de la contrainte équivalente haute $H^{\max }$ (Bourgine, 1982). Il est parfois possible d'admettre une faible contradiction : on dit qu'il y a $\varepsilon$-satisfaction des contraintes discrétisées. Mais si les contraintes ont un caractère absolu, ce qui est rare, il faut réétudier le projet.

\section{Principe du minimum et $\varepsilon$-optimisation}

\subsection{Présentation générale de la méthode d'optimisation}

Soit $h_{0}$ une cote piézométrique donnée à l'origine de l'organe hydraulique comprise dans la plage des contraintes. On peut construire une infinité de lignes piézométriques partant de $h_{0}$ et comprises dans l'entonnoir physique (cf. § 1.2), chacune d'entre elles correspondant à un dimensionnement donné de l'organe.
Parmi toutes les solutions hydrauliques ayant $h_{0}$ comme origine, le principe du minimum permet en théorie de déterminer la ligne piézométrique optimale correspondant à un coût minimal.

L'E-optimisation consiste alors à construire un nombre limité de lignes piézométriques optimales comprises dans l'entonnoir physique dont les cotes amont sont réparties dans la plage de contraintes à l'origine. On retient parmi ces solutions optimales celle qui donne un coût minimal. Cette solution hydraulique n'est pas un optimum absolu. d'où le nom d' $\varepsilon$-optimisation. On approche d'autant mieux l'optimum que le nombre de solutions hydrauliques construites selon le principe du minimum est important.

\subsection{Le principe du minimum}

Soit à résoudre le problème suivant :

Pour un organe hydraulique dont on a construit l'entonnoir physique $E(0-L) \ldots$ Trouver la ligne piézométrique comprise dans $E(0-L)$ et la variable de dimensionnement $u(x)$ appartenant à la gamme technologique $G$ et au domaine de contrainte $D_{x}$ telles que le coût $\mathbf{C}$ de l'organe soit minimal, avec une cote $h(0)$ imposée à l'amont et une cote $h(L)$ libre (cas a) ou imposée (cas b) à l'aval.

Le problème précédent se formalise comme suit :

\section{PROBLEME P}

$$
\underset{u \in G \cap D_{x}}{\operatorname{Min}} \mathbf{C}=\int_{0}^{L} C\{x, z(x), h(x), u\} \cdot d x
$$

avec $h(x) \in E(0-L)$

$$
\begin{aligned}
& \left.\frac{d h}{d x}(x)=g\{x, u(x)\} \quad \text { (équation }\{1.3\}\right) \\
& h(0)=h_{0} \text { imposé } \\
& h(L) \text { libre (cas a) ou } h(L)=h_{L} \text { imposé (cas b). }
\end{aligned}
$$

Le PROBLEME $\mathrm{P}$ entre dans la catégorie des problèmes de commande optimale pour lesquels il existe une terminologie spéciale récapitulée dans l'énoncé suivant :

Trouver pour chaque valeur de $x(0 \leqslant x \leqslant L)$, la commande admissible $u(x)$ (c'est-à-dire appartenant au domaine de commande $G \cap D_{x}$ ) telle que le critère additif $\mathrm{C}$ soit minimal, sachant que la variable d'état $h(x)$ varie selon l'équation d'état $\{1.3\}$ dans le domaine de contrainte sur la variable d'état $E(0-L)$, pour un état initial $h(0)$ imposé et un état final $h(L)$ libre ou imposé.

La variable $u$ est bien une commande dans la mesure où il suffit de connaître sa valeur à chaque abscisse pour pouvoir calculer la valeur de l'état $h$ et du coût $\mathbf{C}$.

La première méthode de résolution d'un problème de commande optimale est la programmation dynamique ou principe de Bellman. Elle a été appliquée avec succès aux projets de réseaux sous-pression, mais elle devient très lourde lorsqu'on veut l'appliquer aux projets de réseaux à surface libre (Ezerzer, 1984).

Nous présentons ici une deuxième méthode connue sous le nom de principe du minimum et qui est basée sur le théorème de Pontriaguine (Pontriaguine, 1960). La SOLUTION S du PROBLEME P se formalise comme suit : 


\section{SOLUTION S}

- Pour qu'il existe un dimensionnement optimal $u(x)$ admissible $\left(u(x) \in G \cap D_{s}\right)$ qui rende minimal le coût $\mathbf{C}$ de l'organe avec $h(x)$ solution de l'équation d'état dans le domaine de contrainte $E(0-L), h(0)=h_{0}$ imposé et $h(L)$ libre (cas a) ou $h(L)=h_{L}$ imposé (cas b),

- Il est nécessaire qu'il existe une fonction auxiliaire $\psi(x)$ définissant la fonction Halmiltonien $\mathbf{H}$ par la relation $\mathbf{H}\{x, z(x), h(x), u, \psi(x)\}$

$$
=C\{x, z(x), h(x), u\}+\psi(x) \cdot g\{x, u\}
$$

telle que, pour tout $x(0 \leqslant x \leqslant L)$, la valeur optimale de $\mathrm{H}$ soit égale à :

$$
\begin{aligned}
& \mathbf{H}\{x, z(x), h(x), u(x), \psi(x)\} \\
& =\operatorname{Min}_{u \in G \cap D_{x}}^{\mathbf{H}}\{x, z(x), h(x), u, \psi(x)\}
\end{aligned}
$$

où $\psi(x)$ est solution de l'équation d'optimalité

$$
\frac{d \psi}{d x}(x)=-\frac{\partial \mathbf{H}}{\partial h}\{x, z(x), h(x), u(x), \psi(x)\}
$$

avec a. $\psi(L)=0$ si $h(L)$ libre

b. $\psi(L)=\psi_{L} \neq 0$ si $h(L)=h_{L}$ imposé.

Pour appliquer le théorème de Pontriaguine, nous avons supposé que les fonctions et les domaines de commande sur $u$ et de contrainte sur $h$ ont les propriétés requises. Mais il faut surtout bien noter qu'il s'agit d'une condition nécessaire. Nous avons de la même façon postule que la condition est suffisante, sans démonstration. La seule validation que nous proposons est faite a posteriori, dans la mesure où la méthode conduit à des résultats satisfaisants.

La variable $\psi$ a une signification bien particulière. On peut démontrer que (Attali, 1981), quelles que soit les conditions sur l'état final (cas a ou cas b), on a :

$$
\psi(x)=+\frac{\partial \mathbf{C}(x)}{\partial h(x)}
$$

avec

$$
\mathbf{C}(x)=\int_{x}^{L} C\{\xi, z(\xi), h(\xi), u(\xi)\} \cdot d \xi
$$

où $\xi$ représente l'abscisse longitudinale.

L'équation $\{2.4\}$ s'applique en particulier à l'état initial $h(0)=h_{0}$. D'où :

$$
\psi(0)=+\frac{\partial \mathbf{C}(0)}{\partial h(0)}=+\frac{\partial \mathbf{C}}{\partial h_{0}}
$$

Les équations $\{2.4\}$ et $\{2.5\}$ montrent que, au voisinage de l'optimum, la variable $\psi$ représente un coût marginal par rapport à la cote piézométrique. $\psi(x)$ représente le coût marginal local du sous-organe compris entre $x$ et $L$ et $\psi(0)$ le coût marginal global de l'organe entier.

\subsection{Technique de résolution du principe du minimum}

Le paragraphe 2.2 présente le théorème de Pontriaguine sous sa forme continue, ce qui suppose une résolution analytique, c'est-à-dire que l'on peut déterminer explicitement les fonctions $u(x)$ et $h(x)$. Ce cas est valable lorsque les fonctions figurant dans les données du problème sont simples, en particulier dans le cas de conduites sous pression avec une variable de commande unidimensionnelle.

En général, par exemple pour les canaux à surface libre (variable de commande bidimensionnelle), on ne peut résoudre les équations différentielles que pas à pas. Il faut alors choisir un pas de calcul $\Delta x$ (en étudiant si possible la sensibilité de ce paramètre) et utiliser un moyen informatique pour la recherche du minimum de la fonction $\mathbf{H}$.

Le calcul d'une ligne piézométrique optimale s'effectue alors en utilisant l'ALGORITHME A décrit ci-dessous.

\section{Algorithme A}

Boucle sur $x$ (cf. figure 4).

$$
\begin{aligned}
& x=L \quad z(L)=z_{L} \text { (donnée) } \\
& h(L)=h_{L} \\
& \psi(L)=0\left(\text { ou } \psi(L)=\psi_{L} \neq 0\right)
\end{aligned}
$$

1. Recherche du minimum de $\mathbf{H}\{x, z(x), h(x), u, \psi(x)\}$ pour $u \in G \cap D_{x}$, les autres variables étant fixées. On en déduit $u(x)$, valeur optimale de la commande $u$ entre $(x-\Delta x)$ et $x$.

2. $h(x-\Delta x)=h(x)+\int_{x}^{x-\Delta x} g\{\xi, u(x)\} \cdot d \xi$

Vérification que : $h(x-\Delta x) \in E(0, L)$. Sinon : $h(x-\Delta x)$ $=H^{\max }(x-\Delta x)$ ou $h(x-\Delta x)=H^{\min }(x-\Delta x)$ selon la contrainte rencontrée.

3. $\psi(x-\Delta x)=$

$\psi(x)-\int_{x}^{x-\Delta x} \frac{\partial \mathbf{H}}{\partial h}\{\xi, z(\xi), h(\xi), u(x), \psi(x)\} \cdot d \xi$

4. Retour à 1 avec $x=x-\Delta x$ ou arrêt si $x=0$.

La ligne piézométrique ainsi obtenue est optimale pour le PROBLEME P dont l'état initial est la cote $h(0)$ calculée par l'ALGORITHME A. On procède par itérations successives sur $h_{L}$ (cas $a$, avec $\psi(L)=0$ ) ou $\psi_{L}$ (cas $b$, avec $h(L)=h_{L}$ ) pour rechercher la ligne optimale correspondant à la cote amont $h_{0}$ imposée.

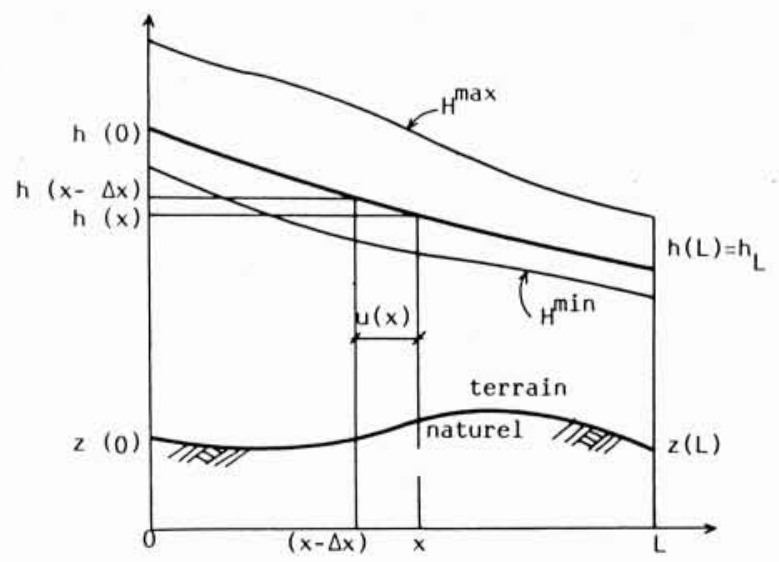

4. Calcul pas à pas d'une ligne piézométrique optimale. 


\subsection{L'E-optimisation}

L'E-optimisation consiste à construire un nombre limité de lignes optimales, mais bien réparties dans l'entonnoir physique de façon à approcher l'optimum absolu avec une précision suffisante.

On peut exposer rapidement une technique permettant d'assurer une bonne répartition des lignes piézométriques optimales dans l'entonnoir (cf. figure 5).

Le projeteur sélectionne tout d'abord un certain nombre de cotes d'extrémité $h_{L}$ et construit les lignes optimales issues de ces points avec la condition $\psi(L)=0$. D'après le principe du minimum, cela signifie que l'on cherche des lignes optimales à extrémité aval "libre ". Certaines d'entre elles peuvent être dégénérées, c'est-à-dire confondues en tout ou partie avec les bords de l'entonnoir physique.

Si toute la plage amont n'est pas explorée, on construit quelques lignes avec $\psi_{L} \neq 0$ à partir des cotes $H^{\min }$ ou $H^{\max }$ à l'aval de l'organe. D'après le principe du minimum, les lignes calculées seront optimales, avec une cote d'extrémité aval imposée.

Il n'est pas nécessaire de construire des lignes "à état final imposé " à partir de cotes différentes de $H^{\min }$ ou $H^{\max }$ compte tenu des propriétés de convexité qui seront mises en évidence par la suite (cf. § 3.2.4 et 4.2.2).

A chaque étape du calcul d'une ligne optimale, les seules variables à conserver sont les valeurs des variables $h, \psi$ et C. Si plusieurs lignes piézométriques ont une cote piézométrique commune à l'amont de l'organe, on ne retient que la ligne de coût minimal.

Parmi toutes les lignes optimales, on ne retient que celle dont le coût est minimal. Les caractéristiques de dimensionnement définitives s'obtiennent par un calcul effectué de l'amont vers l'aval à partir des valeurs intermédiaires de $h, \psi$ et $\mathbf{C}$ pour la ligne retenue, ce qui évite d'occuper une trop grande place dans la mémoire de l'ordinateur.

\section{Dimensionnement d'une rampe en micro-irrigation}

Nous présentons ici un cas sommaire de dimensionnement avec des hypothèses volontairement simplificatrices mais qui ne sont nullement obligatoires pour l'application de la méthode exposée précédemment (Bourgine, 1982; CEMAGREF, 1982).

\subsection{Données}

Soit à dimensionner une rampe en polyéthylène de 100 mètres de longueur $(L)$ équipée de goutteurs d'un même type avec un écartement $(e)$ d'un mètre et délivrant un débit nominal de $4 \mathrm{l} / \mathrm{h}$ sous une charge nominale de 10 mètres. On suppose que le terrain est horizontal, et que la variation de débit tolérée sur toute la rampe est de $10 \%$. Comme la loi débit-pression d'un goutteur est de la forme $q_{g}=K_{g} \cdot h^{\alpha}$, avec $\alpha=0.5$ par exemple, une variation de débit de $10 \%$ correspond à une variation de pression de 2 mètres.

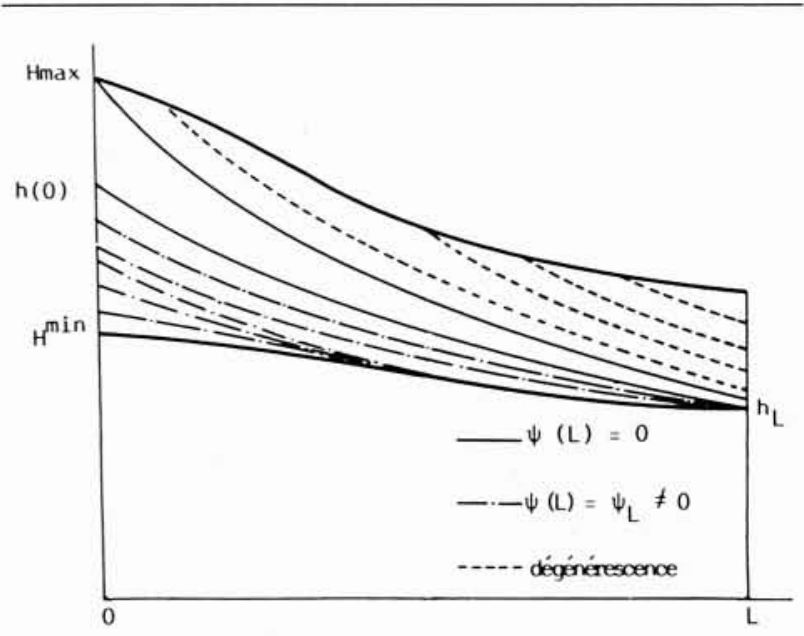

5. Construction des lignes optimales.

On suppose alors en première approximation que tous les goutteurs délivrent le même débit $q_{g}$ de $41 / \mathrm{h}$ si la ligne piézométrique est comprise entre $9 \mathrm{~m}$ et $11 \mathrm{~m}$.

En première approximation, le débit varie de façon continue :

$$
Q(x)=\frac{q}{e} \cdot(L-x) \quad(0 \leqslant x \leqslant L)
$$

où $q$ désigne le débit par unité de longueur $q_{x} / e$

La gamme technologique de rampe est discontinue. Elle est composée de 5 diamètres (diamètres intérieurs exprimés en $\mathrm{mm}$ ) :

$G=\left\{\phi_{1}=13, \phi_{2}=14.5, \phi_{3}=17, \phi_{4}=22, \phi_{5}=28\right\}$ $\{1.1\}$ :

Les contraintes sur la vitesse s'expriment par la relation

$$
D_{x}=\left\{\phi / 0 \leqslant \frac{4}{\pi} \cdot \frac{Q(x)}{\phi^{2}} \leqslant 2,5 \mathrm{~m} / \mathrm{s}\right\}
$$

Pour exprimer le coût $\mathbf{C}$ de la rampe, on suppose que les tuyaux appartiennent à une seule classe de pression et on néglige le coût de mise en place et le coûts des raccordements entre tuyaux de diamètres différents.

L'équation $\{1.2\}$ s'écrit :

$$
\mathbf{C}=\int_{0}^{L} p\{\phi(x)\} \cdot d x
$$

où $p$ désigne le prix au mètre linéaire de tuyau. On retient la formule de prix approchée suivante :

$$
p\left\{\phi_{i}\right\}=(0.007) \cdot \phi_{i}^{2}
$$

avec $\phi_{i}$ en $\mathrm{mm}$ et $p\left\{\phi_{i}\right\}$ en $\mathrm{F} / \mathrm{m}$.

La loi de perte de charge retenue (équation $\{1.3\}$ ) est celle que l'on utilise au CEMAGREF pour les tuyaux en polyéthylène :

$$
\frac{d h}{d x}(x)=-Q^{m}(x) \cdot f\{\phi(x)\}
$$

avec $m=1.75$ et $f\{\phi\}=+(0.478) \cdot \phi^{-4.75}$

$d h / d x$ en $\mathrm{m} / \mathrm{m}, \phi$ en mm et $Q$ en $1 / \mathrm{h}$. 


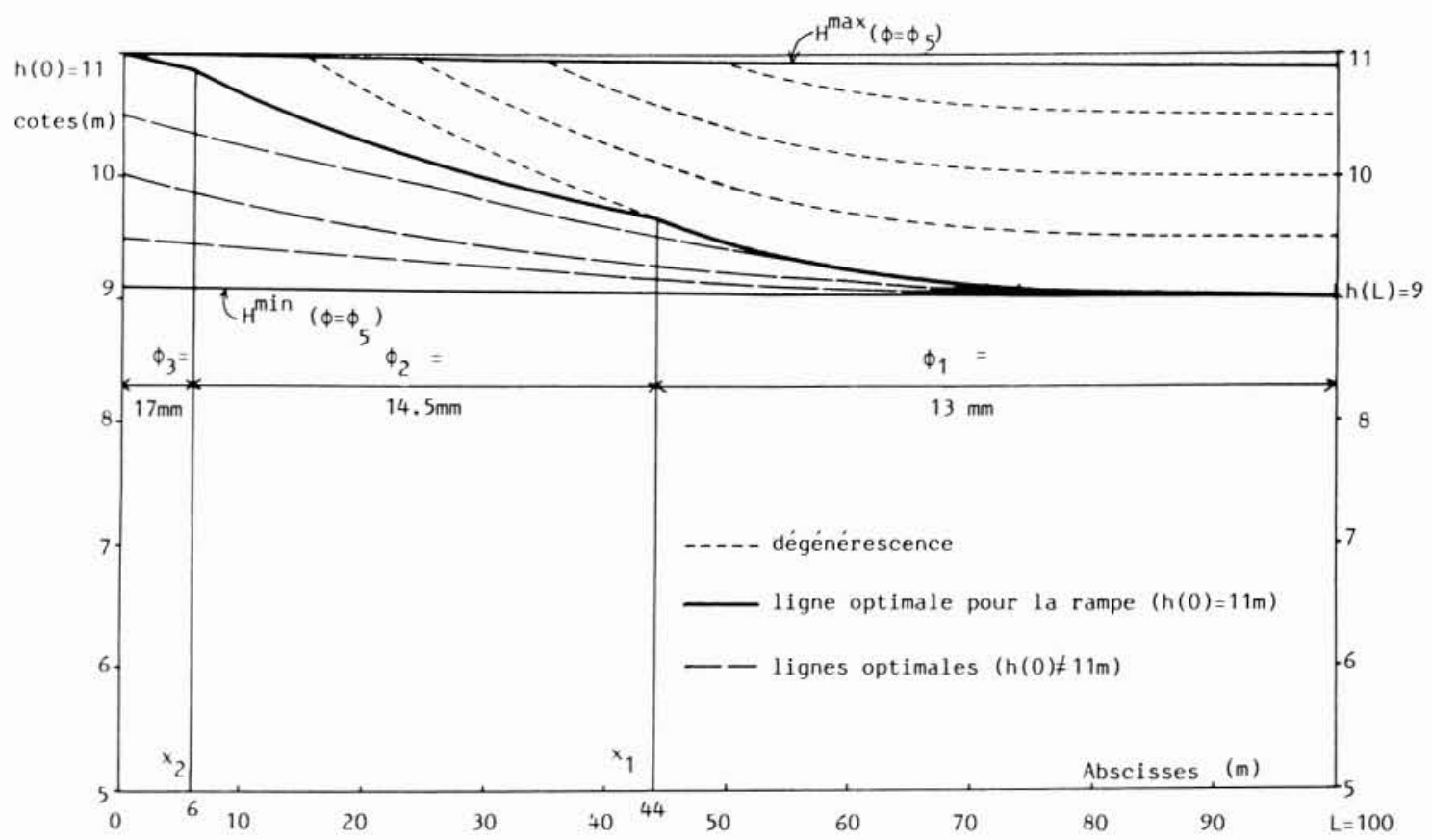

6. E-optimisation sur une rampe de micro-irrigation.

\subsection{Résolution}

\subsubsection{Existence d'une solution hydraulique}

Appliquons la technique de construction de l'entonnoir physique $E(0-L)$ exposée au chapitre 1 .

Les abscisses contraignantes (cf. $\S 1.1 .7$ ) sont 0 et $L$ avec :

$h^{\min }(0)=h^{\min }(L)=9 \mathrm{~m}$ et $h^{\max }(0)=h^{\max }(L)=11 \mathrm{~m}$

Les pentes $I^{\min }$ et $I^{\max }$ (cf. $\S 1.2$ ) correspondent respectivement aux diamètres $\phi_{\mathrm{S}}=28 \mathrm{~mm}$ et $\phi_{1}=13 \mathrm{~mm}$. D'où l'on déduit que les contraintes équivalentes sont les lignes piézométriques calculées avec le daimètre $\phi_{5}$ passant par (cf. figure 6) :

- $h^{\min }(L)=9 \mathrm{~m}$ pour la contrainte équivalente basse,

- $h^{\max }(0)=11 \mathrm{~m}$ pour la contrainte équivalente haute.

\subsubsection{Détermination de lignes optimales}

L'application du principe du minimum conduit à résoudre dans un premier temps un problème de la forme (cf. PROBLEME P, § 2.2.) :

$$
\underset{\phi \in G \cap D_{x}}{\operatorname{Min}} \mathbf{C}=\int_{0}^{L} p\{\phi\} \cdot d x
$$

avec

$$
\frac{d h}{d x}(x)=-Q^{m}(x) \cdot f\{\phi(x)\}
$$

$h(0)=h_{0}$

et $h(L)$ libre (cas a) ou $h(L)=h_{L}$ imposé (cas b).
Quelle que soit la condition sur $h(L)$, on a (cf. SOLUTION S, $\S 2.2$, équations $\{2.1\}$ à $\{2.3\}$ ), sachant que $z(x)$ n'intervient pas dans les fonctions coût et perte de charge :

$\mathbf{H}\{x, h(x), \phi, \psi(x)\}=p\{\phi\}-\psi(x) \cdot Q^{m}(x) \cdot f\{\phi\}$

$\mathbf{H}\{x, h(x), \phi(x), \psi(x)\}=\operatorname{Min}_{\phi \in G \cap D_{x}} \mathbf{H}\{x, h(x), \phi, \psi(x)\}$

$$
\frac{d \psi}{d x}(x)=-\frac{\partial \mathbf{H}}{\partial h}\{x, h(x), \phi(x), \psi(x)\}=0
$$

d'où $\psi(x)=$ cste $(0 \leqslant x \leqslant L)$

\subsubsection{Cote $h(L)$ libre}

$\psi(L)=0$, donc $\psi(x)=0(0 \leqslant x \leqslant L)$

On peut vérifier que le dimensionnement optimal est obtenu pour $\phi_{1}$, mais, avec ce diamètre, la perte de charge sur la longueur $L$ dépasse la plage tolérée $(\Delta h=3,18 \mathrm{~m}>$ $2 \mathrm{~m}$ ).

On obtient donc une solution dégénérée à deux diamètres $\left(\phi_{1}\right.$ et $\left.\phi_{5}\right)$. La figure 6 représente quelques lignes dégénérées issues de différentes cotes $h_{L}$.

\subsubsection{Cote $h(L)=h_{L}$ imposée}

On a alors $\psi(x)=\psi_{L} \neq 0(0 \leqslant x \leqslant L)$

$\mathbf{H}\left\{x, h(x), \phi(x), \psi_{L}\right\}$

$$
=\operatorname{Min}_{\phi \in G \cap D_{x}}\left[p\{\phi\}-\psi_{L} \cdot Q^{m}(x) \cdot f\{\phi\}\right]
$$


Il existe une solution analytique qui peut aussi être mise en évidence par la relaxation Lagrangienne (Bourgine, 1982; Pleban et al., 1984).

Le problème à résoudre consiste à trouver les différents diamètres de la rampe. On peut montrer, ce que nous ne ferons pas ici pour alléger la présentation, que dans l'exemple retenu :

- le dimensionnement optimal correspondant à une valeur de $\psi_{L}$ est une série croissante de diamètres dans le sens des débits croissants (de l'aval vers l'amont);

- la série comprend le plus petit diamètre $\left(\phi_{1}=13 \mathrm{~mm}\right)$, et si un diamètre $\phi_{i}$ fait partie de la série, tous les diamètres intermédiaires entre $\phi_{1}$ et $\phi_{i}$ en font aussi partie.

Soit $j(1 \leqslant j \leqslant 5)$ le nombre de diamètres intervenant dans le dimensionnement optimal. Il s'agit de déterminer la valeur de $j$ et des $(j-1)$ points de raccordement $x_{k}$ entre les diamètres $\phi_{k}$ et $\phi_{k+1}\left(x_{j-1} \leqslant x_{k} \leqslant x_{1}\right)$.

De l'équation $\{3.1\}$ on tire :

$$
\begin{aligned}
p\left\{\phi_{k}\right\}-\psi_{L} \cdot Q^{m}\left(x_{k}\right) & \cdot f\left\{\phi_{k}\right\}= \\
& p\left\{\phi_{k+1}\right\}-\psi_{L} \cdot Q^{m}\left(x_{k}\right) \cdot f\left\{\phi_{k+1}\right\}
\end{aligned}
$$

D’où

avec $\quad \psi_{L}=-\frac{\beta_{k}}{q^{m} \cdot\left(L-x_{k}\right)^{m}}(1 \leqslant k \leqslant j-1)$

$$
\beta_{k}=\frac{p\left\{\phi_{k+1}\right\}-p\left\{\phi_{k}\right\}}{f\left\{\phi_{k}\right\}-f\left\{\phi_{k+1}\right\}}
$$

De plus,

$$
\begin{aligned}
\mathrm{h}_{0}-h_{L} & =\int_{0}^{L} Q^{m}(x) \cdot f\{\phi(x)\} \cdot d x \\
& =\sum_{r=1}^{1} f\left\{\phi_{r}\right\} \cdot \int_{x_{r}}^{x_{r-1}} q^{m} \cdot(L-x)^{m} \cdot d x
\end{aligned}
$$

avec $x_{0}=L$ et $x_{i}=0$

La résolution de ce système d'équations (Bourgine, 1982) consiste tout d'abord à définir :

$$
b=\frac{1}{m+1} \sum_{k=1}^{u-1)} \beta_{k}^{1 / m} \cdot\left[p\left\{\phi_{k+1}\right\}-p\left\{\phi_{k}\right\}\right]
$$

D'où il vient :

$$
\begin{aligned}
& \psi_{L}=-\left[\frac{b / q}{h_{0}-h_{L}-f\{\phi\} \cdot q^{m} \cdot L^{m+1} /(m+1)}\right]^{\frac{m}{m+1}} \\
& \mathbf{C}=p\left\{\phi_{\}} \cdot L-(m+1) \cdot\left(\frac{b}{q}\right)^{\frac{m}{m+1}} .\right. \\
& {\left[h_{0}-h_{L}-f\left\{\phi_{\rangle}\right\} \cdot q^{m} \cdot L^{m+1} /(m+1)\right]^{\frac{1}{m+1}}}
\end{aligned}
$$

Les équations $\{3.2\}$ à $\{3.5\}$ permettent de calculer la ligne piézométrique optimale joignant n'importe quelles cotes $h_{0}$ et $h_{L}$ comprises dans l'entonnoir physique $E(0-L)$.

L'équation $\{3.6\}$ montre que :

$$
\frac{\partial \mathbf{C}}{\partial h_{L}}=-\psi_{L} \geqslant 0 \quad \text { et } \quad \frac{\partial \mathbf{C}}{\partial h_{0}}=+\psi_{L} \leqslant 0
$$

Par conséquent, le coût minimal est obtenu pour :

$$
h_{0}=11 \mathrm{~m} \quad \text { et } \quad h_{L}=9 \mathrm{~m}
$$

Dans ce dernier cas, la résolution analytique des équations $\{3.2\}$ à $\{3.6\}$ conduit à une rampe à 3 diamètres $\left(\phi_{1}=13 \mathrm{~mm}, \phi_{2}=14.5 \mathrm{~mm}\right.$ et $\phi_{3}=17 \mathrm{~mm}$ ), avec (cf. figure 6):

$$
x_{1}=44 \mathrm{~m}, x_{2}=6 \mathrm{~m} \quad \text { et } \quad \mathrm{C}=134 \mathrm{~F}
$$

\subsection{Critique de la méthode}

Les hypothèses simplificatrices de l'exemple traité cidessus peuvent être pratiquement toutes levées. On peut en particulier introduire des coûts supplémentaires de raccords ou prendre en compte la loi débit-pression des goutteurs dans la formulation théorique pour éviter d'avoir recours à l'approximation sur l'uniformité des débits lorsque la pression est comprise entre $9 \mathrm{~m}$ et $11 \mathrm{~m}$. Cette dernière modification présente cependant peu d'intérêt car, dans le cas présent, l'erreur sur le débit en tête calculée a posteriori est de l'ordre de $2 \%$.

\section{Dimensionnement d'un canal à surface libre}

\subsection{Données}

Soit à dimensionner un canal trapézoïdal revêtu, dont le tracé en plan est fixé, transitant un débit constant $Q$. sur un terrain naturel horizontal, avec les caractéristiques principales suivantes :

$\begin{array}{ll}\text { Longueur } & L=500 \mathrm{~m} \\ \text { Cote du T.N. } & z(x)=z=200 \mathrm{~m}(0 \leqslant x \leqslant L) \\ \text { Débit } & Q(x)=Q=0.5 \mathrm{~m}^{3} / \mathrm{s}(0 \leqslant x \leqslant L) \\ \text { Fruit intérieur du canal } & a=1 \text { (cf. figure } 1 \text { ) } \\ \text { Coefficient de Strickler } & K_{s}=60\end{array}$

La gamme technologique est continue :

$G=\{(l, t) / 0.3 \mathrm{~m} \leqslant l \leqslant 3.0 \mathrm{~m}, 0.1 \mathrm{~m} \leqslant t \leqslant 3.0 \mathrm{~m}\}$ $\{1.1\}$ :

Les contraintes sur la vitesse s'expriment par la relation

$$
D_{x}=\left\{(l, t) / 0.5 \mathrm{~m} / \mathrm{s} \leqslant \frac{Q}{(l+a . t) \cdot \mathrm{t}} \leqslant 2.5 \mathrm{~m} / \mathrm{s}\right\}
$$

On suppose que les prix intervenant dans le coût unitaire $C$ (cf. $\S 1.1 .5$, équation $\{1.2\}$ ) sont constants et indépendants de l'abscisse (les prix indiqués n'ont qu'une valeur relative),

remblai

déblai

$$
\begin{aligned}
& \pi_{1}=20 \mathrm{~F} / \mathrm{m}^{3} \\
& \pi_{2}=10 \mathrm{~F} / \mathrm{m}^{3} \\
& \pi_{3}=1 \mathrm{~F} / \mathrm{m}^{2} \\
& \pi_{4}=50 \mathrm{~F} / \mathrm{m}^{2}
\end{aligned}
$$

revêtement

et qu'ils s'appliquent respectivement aux fonctions représentatives de la section de remblai $\left(S_{R B}\right)$, de la section de déblai $\left(S_{D B}\right)$, de la largeur d'emprise $\left(L_{D E}\right)$ et de la largeur de revêtement $\left(L_{R V}\right)$. On n'explicitera pas ces différentes fonctions (Ezerzer, 1984). 
On néglige le coût des raccordements aux changements de section.

$$
\mathbf{C}=\int_{0}^{t} C\{x, z(x), h(x), l(x), t(x)\} \cdot d x
$$

avec :

$$
C=\pi_{1} \cdot S_{R B}+\pi_{2} \cdot S_{D B}+\pi_{3} \cdot L_{D E}+\pi_{4} \cdot L_{R V}
$$

Enfin, la loi de perte de charge (équation $\{1.3\}$ ) est la formule de Manning-Strickler :

$$
\frac{d h}{d x}(x)=-Q^{m} \cdot f\{l(x), t(x)\}
$$

avec :

$m=2$

$$
\begin{aligned}
& \text { et } f\{l, t\}=+\frac{1}{K_{s}^{2}} \cdot \frac{\left[\{l+2\} \cdot\left(1+a^{2}\right)^{1 / 2} \cdot t\right]^{4 / 3}}{[(l+a \cdot t) \cdot t]^{10 / 3}} \\
& \frac{d h}{d x} \text { en } \mathrm{m} / \mathrm{m}, Q \text { en } \mathrm{m}^{3} / \mathrm{s}, l \text { et } t \text { en } m
\end{aligned}
$$

\subsection{Résolution}

\subsubsection{Calcul des lignes piézométriques optimales}

Il s'agit dans un premier temps de résoudre un problème de la forme (cf. PROBLEME P, § 2.2) :

$$
\underset{(l, t) \in G \cap D_{x}}{\operatorname{Min}} \mathbf{C}=\int_{0}^{L} C\{x, z(x), h(x), l, t\} \cdot d x
$$

avec :

$$
\begin{gathered}
\frac{d h}{d x}(x)=-Q^{m} \cdot f\{l(x), t(x)\} \\
h(0)=h_{0}
\end{gathered}
$$

et $h(\mathrm{~L})$ libre (cas a) ou $h(L)=h_{L}$ imposé (cas b).

La résolution passe par la mise en œuvre de l'algorithme défini précédemment (ALGORITHME A, § 2.3), car, dans le cas présent, il n'y a pas de solution analytique. A chaque étape de calcul d'une ligne piézométrique optimale, la recherche du minimum de la fonction Hamiltonien avec contraintes sur la variable de dimensionnement ne peut se faire que par l'utilisation d'un programme informatique puissant (Ezerzer, 1984).

Nous ne détaillerons pas l'algorithme de calcul des lignes piézométriques optimales. Il est plus intéressant par contre de montrer les variations du coût optimal du canal en fonction des cotes $h(0)$ et $h(L)$.

\subsubsection{Courbes de coût du canal en fonction des cotes d'extrémité}

Le traitement informatique des données précédentes a permis de calculer point par point les courbes de coût représentées à la figure 7 , avec un pas de calcul $\Delta x=100 \mathrm{~m}$. Chacune des quatre courbes correspond à une cote aval $h(L)=h_{L}$ imposée et représente la variation $d u$ coût en fonction de la cote amont $h(0)$. On n'a pas représenté les courbes complètes qui sont en réalité toutes limitées à gauche et à droite, compte tenu des contraintes sur $l$ et $t$ (Ezerzer, 1984).

Remarquons tout d'abord que ces courbes présentent un minimum et ont une allure convexe. S'il est intéressant de démontrer que cette propriété est générale (ce qui n'est pas de notre compétence), il n'en demeure pas moins essentiel de savoir qu'elle se vérifie au moins dans certains cas pratiques, ce qui n'était pas évident a priori. Notons aussi que, pour une cote $h(0)$ fixée, la ligne piézométrique optimale ne correspond pas nécessairement à la cote $h(L)$ la plus faible. On obtient des courbes analogues d'allure convexe en permutant $h(0)$ et $h(L)$.

S'il n'y a pas de contraintes sur les cotes $h(0)$ et $h(L)$, on choisit le point de coût minimal sur l'ensemble des courbes établies. Il s'agit en l'occurence de la ligne piézométrique dont les caractéristiques sont les suivantes :
$h(0)=200.60 \mathrm{~m}$
$h(L)=199.00 \mathrm{~m}$
C $\quad=76291 \mathrm{~F}$

S'il existe des contraintes sur les cotes $h(0)$ et $h(L)$, on choisit le point de coût minimal respectant ces contraintes. Mentionnons à ce propos le cas important en irrigation où l'on veut que la ligne piézométrique domine le terrain naturel, donc la cote $200 \mathrm{~m}$ dans l'exemple choisi. D'après la figure 7 , le point de coût minimal respectant cette contrainte correspond au minimum de la courbe 200 , soit :
$h(0)=200.90 \mathrm{~m}$
$h(L)=200.00 \mathrm{~m}$
C $\quad=86288 \mathrm{~F}$

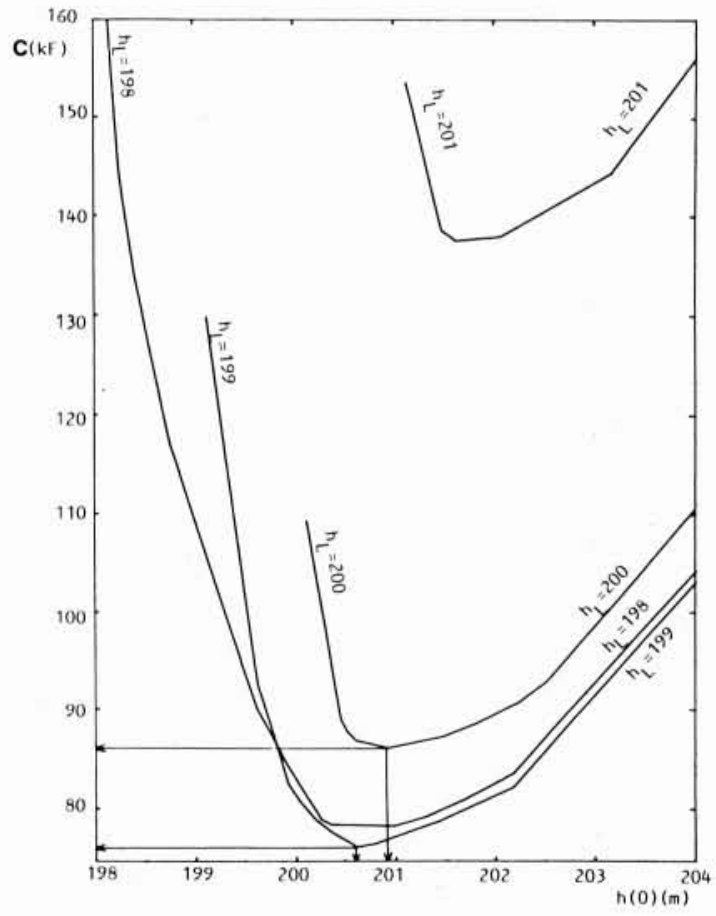

7. Courbes de coût d'un canal en fonction de la cote amont, pour quatre cotes aval imposées. 


\subsection{Critique de la méthode}

Il est possible d'amender la méthode exposée ci-dessus pour l'adapter à des cas plus complexes :

- prise en compte des coûts des changements de section, - introduction de caractéristiques géométriques supplémentaires dans la variable de dimensionnement,

- utilisation d'une gamme discontinue,

- introduction de chutes intermédiaires, à condition que leur emplacement soit décidé par le projeteur, - prise en compte des pertes de charge locales introduites par les changements de section.

Néanmoins, la principale objection concerne les prix. On a supposé en effet que les prix sont constants et connus du projeteur. Or, dans la plupart des cas, on utilise les déblais excédentaires pour les tronçons du canal qui sont en remblai. Comme la part prépondérante du prix des remblais est égale au coût du transport appliqué à des volumes qui ne sont connus qu'après calcul, on ne peut pas décider a priori d'un prix unitaire au $\mathrm{m}^{3}$ de remblais $\left(\pi_{1}\right)$.

La méthode s'applique donc telle quelle lorsque la totalité du remblai doit être apportée de l'extérieur (en faisant éventuellement varier les prix $\pi_{1}$ en fonction de l'abscisse pour tenir compte de l'éloignement des zones de prélèvement), ou bien lorsqu'il n'y a pas de remblai. Il faut procéder par approximations successives sur $\pi_{1}$ et $\pi_{2}$ dans les autres cas.

\section{Conclusion}

Moyennant quelques améliorations et peut-être quelques simplifications, la méthode d'optimisation que l'on vient de présenter semble donc utilisable pour le dimensionnement d'un réseau arborescent quelconque : tuyau sous pression ou canal à surface libre, gamme technologique discrète ou continue, débit variant par saut ou de façon graduelle, tous ces cas sont passibles de cette même formalisation.

Cette méthode ne s'oppose pas aux méthodes optimales existantes d'élaboration des projets. Elle vise d'abord à apporter une solution optimale approchée lorsque les méthodes optimales exactes sont très coûteuses, voire inaccessibles, comme pour les projets de micro-irrigation ou de canaux à surface libre.

Mais surtout, elle pourrait être la méthode générale pour une $\varepsilon$-optimisation des avant-projets, c'est-à-dire une méthode améliorante par rapport à une solution initiale mais ne garantissant pas l'optimum global. Elle permettrait alors des évaluations rapides mais approchées d'une bonne solution, et devrait être d'une grande utilité dans tout système d'ingéniérie assistée par ordinateur (SIAO) destiné à la conception de réseaux arborescents de transport de l'eau.

\section{Références}

[1] Attal J. - Analyse économique de la vie politique, PUF. 1981. pp. $184-188$.

[2] Bourgine P. - Réseaux hiérarchisés de transport de l'eau. CEMAGREF, Groupement d'Aix-en-Provence, Division Irrigation. 1982 (48 pages).

[3] CEMAGREF (Division Irrigation). - Un logiciel pour le calcul des installations parcellaires de micro-irrigation. Informations techniques du CEMAGREF, Cahier 46, $\mathrm{n}^{\circ}$ 6. Juin 1982. (6 pages).

[4] CEMAGREF (Division Hydraulique Générale). - Calcul des réseaux ramifiés sous pression. Etude de CEMAGREF, $\mathrm{n}^{\circ} 506$. Décembre 1983 (120 pages).

[5] Ezerzer M. - Calcul optimisé d'un réseau d'irrigation à surface libre. Dimensionnement des canaux. Mémoire de $3^{\mathrm{e}}$ année de l'ENITRTS. CEMAGREF, Groupement d'Aix-en-Provence, Division Irrigation. Novembre 1984 (140 pages).
[6] Labye Y. et Lechapt G. - Méthodes permettant de déterminer les caractéristiques optimales d'un réseau de distribution d'eau. Bulletin technique du Génie rural, n 50. Avril 1961 (120 pages).

[7] Legras J. - Algorithmes et programmes d'optimisation non linéaire avec contraintes. Application au contröle optimal. Masson. 1979 (188 pages).

[8] Pleban S., Shacham D. et Loftis J. - Minimizing capital costs of multi-outlet pipelines (Recherche du coût d'investissement minimal des conduites avec service en route). Journal of Irrigation and Drainage Engineering, Vol. $110, \mathrm{n}^{\circ} 2$. June 1984 (pp. 165-178).

[9] Pontriaguine L., Boltianski V., Gamkrelidze K. et Micht. CHENKO E. - Théorie mathématique des processus optimaux.

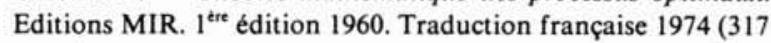
pages). 